\title{
CA-125 Significance in Cirrhosis and Correlation with Disease Severity and Portal Hypertension
}

\section{Julio Collazos*}

\section{Infectious Diseases Unit, Hospital de Galdácano-Usánsolo, Vizcaya, Spain}

*Correspondence to: Julio Collazos, Infectious Diseases Unit, Hospital de Galdácano-Usánsolo, Herriko Gudarien 11, Durango (Vizcaya) 48200, Spain Tel/Fax: +34-94-603-2867, E-mail: med003033@gmail.com

Edula et al. ${ }^{1}$ in their retrospective study of 172 patients concluded that serum concentrations of cancer antigen-125 (CA-125) are elevated in cirrhotic patients with ascites. This relationship has been well known for several decades, ${ }^{2-6}$ as well as the relationship of CA-125 with pleural and pericardial effusions, among many other non-malignant conditions, ${ }^{4,5,7}$ because $\mathrm{CA}-125$ is expressed in the coelomic epithelium. ${ }^{8}$ In fact, ascitic fluid concentrations of CA-125 are even higher than in serum. ${ }^{2}$

Edula et al. ${ }^{1}$ said that cirrhotic patients without ascites had normal mean CA-125 concentrations, and define this observation as "a new finding". However, these observations are not new. More than 25 years ago our group published a prospective and comprehensive study of 159 patients with liver diseases, including 85 cirrhotics with and without ascites. ${ }^{3}$ In this article, cited by Edula et al. as their reference 14, we found the same results as Edula et al. ${ }^{1}$ reported for ascitic and non-ascitic patients. ${ }^{1}$ Moreover, our group published other articles focused exclusively on patients without ascites.9,10 Like Edula et al., ${ }^{1}$ we found that elevations of CA-125 concentrations in patients without ascites were uncommon, but also that these elevations were related to the degree of liver dysfunction.

Therefore, CA-125 increases markedly (even 100 times above the upper normal level) in patients with ascites, an increase that we previously found to be highly proportional to the amount of ascites as measured semiquantitatively in 5 degrees, and it decreases rapidly with the diminution of ascites. In fact, CA-125 proved to be a reliable marker of ascites (sensitivity $98.4 \%$, specificity $95.9 \%$, positive predictive value $93.8 \%$, negative predictive value $98.9 \%$, efficiency $96.9 \%)^{3}$

Although to a considerably lower degree, and much more infrequently, we also previously found increased CA-125 concentrations in some patients who did not have ascites, as evaluated ultrasonographically, as well as significant correlations between CA-125 and some liver function markers, such as albumin and prothrombin. ${ }^{9}$ Our findings suggested that, apart from the determinant role of ascites, liver dysfunction itself is also responsible for moderate increases in the serum concentrations of CA-125, probably due to a poor metabolization of this glycoprotein. ${ }^{9,10}$

On the other hand, the absence of statistical significance regarding portal hypertension $(p=0.1)$ reported by Edula et al. ${ }^{1}$ is surprising, considering that the differences in the CA-125 concentrations that they found were substantial (414 vs. $256 \mathrm{U} / \mathrm{mL}$ ) and that portal hypertension is tightly related to ascites. The authors evaluated portal hypertension by a history of esophageal varices. Besides the limited sensitivity of this method to detect portal hypertension, perhaps the number of patients studied was too small (Type II error). Also, the parametric tests used by the authors for statistical calculations ( $t$-test, ANOVA) might have been inappropriate, as we found that the distribution of CA-125 was markedly non-Gaussian, and the illustrations of Edula et al. ${ }^{1}$ suggest the same.

In our series, we found marked differences in CA-125 concentrations in patients with or without portal hypertension, as evaluated separately by three different methods: echography, esophageal varices and splenomegaly $(p<$ 0.0001 for each). ${ }^{3}$ Even portal hypertension was associated with higher $\mathrm{CA}-125$ concentrations in patients without ascites $(p=0.049){ }^{9}$

Citation of this article: Collazos J. CA-125 significance in cirrhosis and correlation with disease severity and portal hypertension. J Clin Transl Hepatol 2018;6(4):447-448. doi: 10. 14218/JCTH.2018.00056.

\section{Conflict of interest}

The author has no conflict of interest related to this publication.

\section{References}

[1] Edula RG, Muthukuru S, Moroianu S, Wang Y, Lingiah V, Fung P, et al. CA-125 significance in cirrhosis and correlation with disease severity and portal hypertension: A retrospective study. J Clin Transl Hepatol 2018;6:241246. doi: $10.14218 /$ JCTH.2017.00070.

[2] Bergmann JF, Bidart JM, George M, Beaugrand M, Levy VG, Bohuon C. Elevation of CA 125 in patients with benign and malignant ascites. Cancer 1987;59:213-217. doi: 10.1002/1097-0142(19870115)59:2<213::AID-CNCR2820590206>3.0.CO:2-I

[3] Collazos J, Genolla J, Ruibal A. CA 125 serum levels in patients with nonneoplastic liver diseases. A clinical and laboratory study. Scand J Clin Lab Invest 1992;52:201-206. doi: 10.3109/00365519209088786.

[4] Eerdekens MW, Nouwen EJ, Pollet DE, Briers TW, De Broe ME. Placental alkaline phosphatase and cancer antigen 125 in sera of patients with benign and malignant diseases. Clin Chem 1985;31:687-690.

[5] Mezger J, Wilmanns W, Lamerz R. Elevated serum CA 125 levels in patients with benign ascitic or pleural effusions. Tumour Biol 1988;9:47-52. doi: $10.1159 / 000217544$.

[6] Bergmann JF, Beaugrand M, Labadie H, Bidart JM, Bohuon C. CA 125 (ovarian tumour-associated antigen) in ascitic liver diseases. Clin Chim Acta 1986; 155:163-165. doi: 10.1016/0009-8981(86)90278-0.

[7] Touitou Y, Bogdan A. Tumor markers in non-malignant diseases. Eur ] Cancer Clin Oncol 1988;24:1083-1091.

[8] Kabawat SE, Bast RC Jr, Bhan AK, Welch WR, Knapp RC, Colvin RB. Tissue distribution of a coelomic-epithelium-related antigen recognized by the mono-clonal antibody OC125. Int J Gynecol Pathol 1983;2:275-285. doi: 10.1097/ 00004347-198303000-00005.

[9] Collazos J, Genolla J, Allende MT, Ruibal A. Serum CA 125 levels in patients with non-malignant liver diseases without ascites. Clin Investig 1993; 71:239. doi: 10.1007/bf00180108.

[10] Ruibal A, Siuriana R. Evidence of a relationship between high serum Ca 125 and liver failure pattern in cirrhotic patients without ascitis and jaundice. Int J Biol Markers 1986;1:55-56. doi: 10.1177/172460088600100109. 
The Author's Reply

\section{Raja GR Edula*}

Gastroenterology Clinic, Valley Medical Center, Renton, WA, USA

*Correspondence to: Raja GR Edula, Gastroenterology Clinic, Valley Medical Center, M/S TAL-500, 4011 Talbot Road South, Suite 500, Renton, WA 98005, USA Tel: +1-425-690-3488, Fax: +1-425-690-9088, E-mail: rajreddy14@hotmail.com

We would like to thank Julio Collazos for taking the time to read and critique our study. While we understand that there were several studies done in the past on the same subject, our attempt was to revive interest in the same subject as CA-125 antigen is no longer recommended as part of the diagnostic work-up in patients with ascites as per the most recent American Association for the Study of Liver Diseases (AASLD) guidelines for ascites in cirrhosis. ${ }^{1}$ The findings of our study did lead us to comment that CA-125 could be a useful test, especially in patients with truncal obesity where clinical quantification of ascites could be challenging, and will require radiological imaging which may be more expensive and inconvenient for patients, especially when they are being followed for dose titration of diuretics. ${ }^{2}$

Our study did correlate CA-125 antigen with ascites and the model for end-stage liver disease (MELD) score in a linear regression model and concluded that there was a better statistically significant correlation with ascites as compared to the MELD score. Since the MELD score is currently used as the best objective marker for decompensation in patients with end-stage liver disease, we feel this is a significant finding in our study. This also applies to the albumin-bilirubin (ALBI) score, which is a relatively new 'kid on the block' in the evaluation of patients with cirrhosis. These findings are new and have not been reported from other previous studies.
Since our study was retrospective and relied on available data, we used esophageal varices as a marker of portal hypertension rather than other parameters. Moreover, the elevated total CA-125 in both groups of patients with and without esophageal varices (414 vs. $256 \mathrm{IU} / \mathrm{mL}$ ) was significant, although the significance did not reach the threshold set for statistical significance; this finding was confounded by the fact that several of the patients in both groups had ascites from decompensated cirrhosis, which had the best correlation with elevated CA-125 antigen.

While we understand the limitations of a retrospective study and the probability of a Type 2 error, we were able to conclude that patients with cirrhosis but without ascites had normal values of CA-125 antigen, unlike in the study by Collazos et al., ${ }^{3}$ who concluded that findings of elevated antigen were uncommon in the absence of ascites; moreover, their study concluded that CA-125 antigen was invalidate as a tumor marker. I would also like to highlight that our group of patients studied was homogenous and consisted of patients with cirrhosis exclusively.

\section{Conflict of interest}

The author has no conflict of interest related to this publication.

\section{References}

[1] Runyon BA. Introduction to the revised American Association for the Study of Liver Diseases Practice Guideline management of adult patients with ascites due to cirrhosis 2012. Hepatology 2013;57:1651-1653. doi: 10.1002/hep.26359.

[2] Edula RG, Muthukuru S, Moroianu S, Wang $Y$, Lingiah V, Fung $P$, et al. CA-125 significance in cirrhosis and correlation with disease severity and porta hypertension: A retrospective study. J Clin Transl Hepatol 2018;6:241-246. doi: 10.14218/JCTH.2017.00070.

[3] Collazos J, Genolla J, Ruibal A. CA 125 serum levels in patients with nonneoplastic liver diseases. A clinical and laboratory study. Scand J Clin Lab Invest 1992;52:201-206. doi: 10.3109/00365519209088786. 